\title{
Paclobutrazol biodegradation in unsaturated soil in the Semi-Arid Northeast of Brazil
}

\author{
Fernanda L. Vaz ${ }^{1}$, Thaís A. Gomes ${ }^{1}$, Caio C. A. Rocha ${ }^{1}$, Maria Aparecida Mouco ${ }^{2}$ and \\ Ester R. Gouveia ${ }^{1 *}$ \\ ${ }^{1}$ Department of Antibiotics, Federal University of Pernambuco, CEP: 50670-901, Recife-PE, Brazil. \\ ${ }^{2}$ Brazilian Agricultural Research Corporation, CEP: 56302-970, Petrolina-PE, Brazil.
}

Received 24 April, 2014; Accepted 19 January, 2015

\begin{abstract}
Paclobutrazol (PBZ) is a plant growth regulator, increasing flowe ring and yield that is widely used in mango cultivation in the semi-arid northeastern Brazil. PBZ remains active in the soil for several years. However, it can severely affect the growth and development of subsequent crops, mainly by reducing vegetative vigor. The aim of this study was to investigate PBZ biodegradation in four samples of soil: $P$ G (with PBZ application history and with addition of glycerol); NP-G (without PBZ application history and with addition of glycerol); P-NG (with PBZ application history and without addition of glycerol) and NP-NG (without PBZ application history and without addition of glycerol). The biodegradation experiments were carried out in $125 \mathrm{ml}$ flasks containing $10 \mathrm{~g}$ soil, at room temperature for 63 days. Mathematical models to analyze the kinetics of degradation of PBZ were applied. PBZ residue was less than $1 \%$ in soils with a history, regardless the addition of glycerol. The three models (first-order kinetics, double first-order kinetics and logistic) were well adjusted in these cases (P-G and P-NG). On the other hand, PBZ biodegradation in soil NP-G and NP-NG was $64 \%$, and followed the model of double kinetic. PBZ biodegradation in soil with history was successful, probably because the native microbial had adapted to local environmental conditions.
\end{abstract}

Key words: Paclobutrazol, biodegradation, mathematical models.

\section{INTRODUCTION}

Paclobutrazol (PBZ), a derivative of the triazole group, is now commercially used in many tropical and subtropical fruit crops for regulation of growth, flowering and yield and commonly (Srivastav et al., 2010) used in mango cultivation in the semi-arid northeastern Brazil. However, PBZ remains active in soil for several years and can severely affect the growth and development of subsequent crops, mainly by reducing vegetative vigor (Jackson et al., 1996). Through biodegradation, chemical compounds may be removed or reduced in the soil, but the process is expensive. In this process, the introduction of additional nutrient sources enhances microbial degradation (China et al., 2004).

Mathematical models can help measure high levels of

${ }^{*}$ Corresponding author. E-mail: estergouveia@gmail.com. Tel: +55 8121268949.

Author(s) agree that this article remain permanently open access under the terms of the Creative Commons Attribution License $\underline{4.0 \text { International License }}$ 
toxic substances in the soil, or in the fruit of plants treated with pesticides, and may indicate when such substances should be systematically monitored. Mathematical models are available for various agricultural purposes, among which is the simulation of absorption of organic substances by plants (Fujisawa et al., 2002), as well as the behavior of organic substances in the soil (Gang et al., 2003; Milfont et al., 2008).

The first-order kinetic model has been widely used to describe the kinetics of dissipation of various herbicides found in the soil under field conditions, or in the laboratory (Martins and Mermoud, 1998; López-Galindo et al., 2010). However, for many complex compounds, kinetics first order is not appropriate because the biodegradation of these compounds can occur at different rates throughout the process. In this case, the kinetic model results, using double first-order, have shown to work well, especially when the biodegradation occurs primarily in a fast reaction and then decays at a slow rate (López-Galindo et al., 2010).

Vaz et al. (2012) made a study of PBZ biodegradation in saturated soil without the addition of microorganisms (or only with the participation of microorganisms naturally present in the ground). These authors achieved further adjustments in modeling of PBZ biodegradation using double first-order kinetics.

The aim of the present study was the modeling of biodegradation of PBZ in unsaturated soil in the semi-arid Northeast of Brazil with and without history of application.

\section{MATERIALS AND METHODS}

\section{Soil}

Soil samples used in this study were collected in January, 2012, from a farm located in Petrolina (PE), Brazil. The soil has a sandy texture and is classified as Quartzipsamments Neosoil. This region had been consecutively treated with PBZ for six years (once a year), with an average dose of $3.57 \mathrm{~g}$ of active ingredient per plant. The soil samples were collected 30 days after the last application. An average of $1.5 \mathrm{~kg}$ of soil at a depth of 15 and $30 \mathrm{~cm}$ was collected from four points around eight plants. These samples were stored in a refrigerator until the beginning of the biodegradation tests. The samples without PBZ application history were also taken from the same farm.

\section{Paclobutrazol}

PBZ used for quantification was obtained from Sigma Aldrich (St. Louis, MO, USA). The commercial product (Cultar 25 SC), containing $25 \%$ of the active compound, was used in biodegradation experiments.

\section{Experiments of biodegradation}

Experiments of biodegradation were conducted with the collected soil samples. To each $10 \mathrm{~g}$ of soil with (P-G, P-NG) and without (NP-G, NP-NG) PBZ application history, $30 \mu \mathrm{g} / \mathrm{g}$ PBZ were added from a solution prepared with the commercial product (Cultar 25
SC). The experiments were carried out in $125 \mathrm{ml}$ flasks at room temperature, without stirring for 63 days and in triplicate. Samples were withdrawn after $0,7,14,21,35,49$ and 63 days for the quantification of native microbial and residual PBZ. In experiments with the addition of glycerol (P-G, NP-G), the concentration of this compound in the soil was $150 \mu \mathrm{g} / \mathrm{g}$. Micro-organisms were not added to the soil.

\section{Paclobutrazol quantification}

To each $10 \mathrm{~g}$ of soil, $10 \mathrm{ml}$ of methanol (HPLC grade, Mallinckrodt Baker - Phillipsburg, NJ, USA) was added. This suspension was subjected to vacuum filtration, using a membrane of $0.45 \mu \mathrm{m}$. The filtrate was analyzed by high performance liquid chromatography (HPLC) using an Agilent chromatograph (HP 1100). The chromatographic conditions were: methanol/water (80/20), 0.4 $\mathrm{mL} / \mathrm{min}, 30^{\circ} \mathrm{C}$, and $\mathrm{C}-18$ column (Kinetex; Phenomenex).

\section{Growth quantification}

The native microbial was quantified by the method of counting viable cells. Samples of $1 \mathrm{~g}$ of soil were diluted in sterile water, and plated on Petri dishes with the Tryptone Soy Agar (TSA) culture medium, and incubated for $48 \mathrm{~h}$ at $30^{\circ} \mathrm{C}$. The results were expressed as colony forming unit per milliliter (CFU/ml).

\section{Kinetic modeling}

Three mathematical models (Table 1) were applied to kinetic modeling of PBZ biodegradation: first-order kinetic equation, double first-order kinetics equation and logistic equation (Martins and Mermoud, 1998; Varela et al., 2002; Gang et al., 2003). The models were applied using Sigma Plot 11.0. Analysis of variance was performed using Microcal Origin 6.0 (Table 1).

\section{RESULTS AND DISCUSSION}

Figure 1 shows the PBZ biodegradation kinetics in soil without and with application history, with and without addition of glycerol. Experiments in P-G and P-NG soil showed a sustained reduction after the 14 th day and only around $1 \%$ PBZ remained in the 63th day. This ability of the native microbiota to degrade paclobutrazol was probably due to the historical application. After repeated applications of some pesticides, the microorganisms of the soil can degrade these compounds as they become suited for agrochemical use as a source of carbon for energy production and growth. Although there are some other factors affecting the persistence of agrochemicals in the soil, such as temperature, $\mathrm{pH}$ of the soil, chemical hydrolysis and the water content of the soil, microorganisms seem to play an important role in the degradation of these compounds (Abdelrahman, 2004) (Figure 1).

The residue PBZ in soil without application history of PBZ, containing glycerol (NP-G) or not (NP-NG), was approximately $64 \%$ with 14 days. The lower biodegradation rate in NP-G and NP-NG was due to microbial not being adapted to PBZ, since this soil had no history of application. This rate remained constant probably 
Table 1. Kinetic models for the biodegradation of substrates.

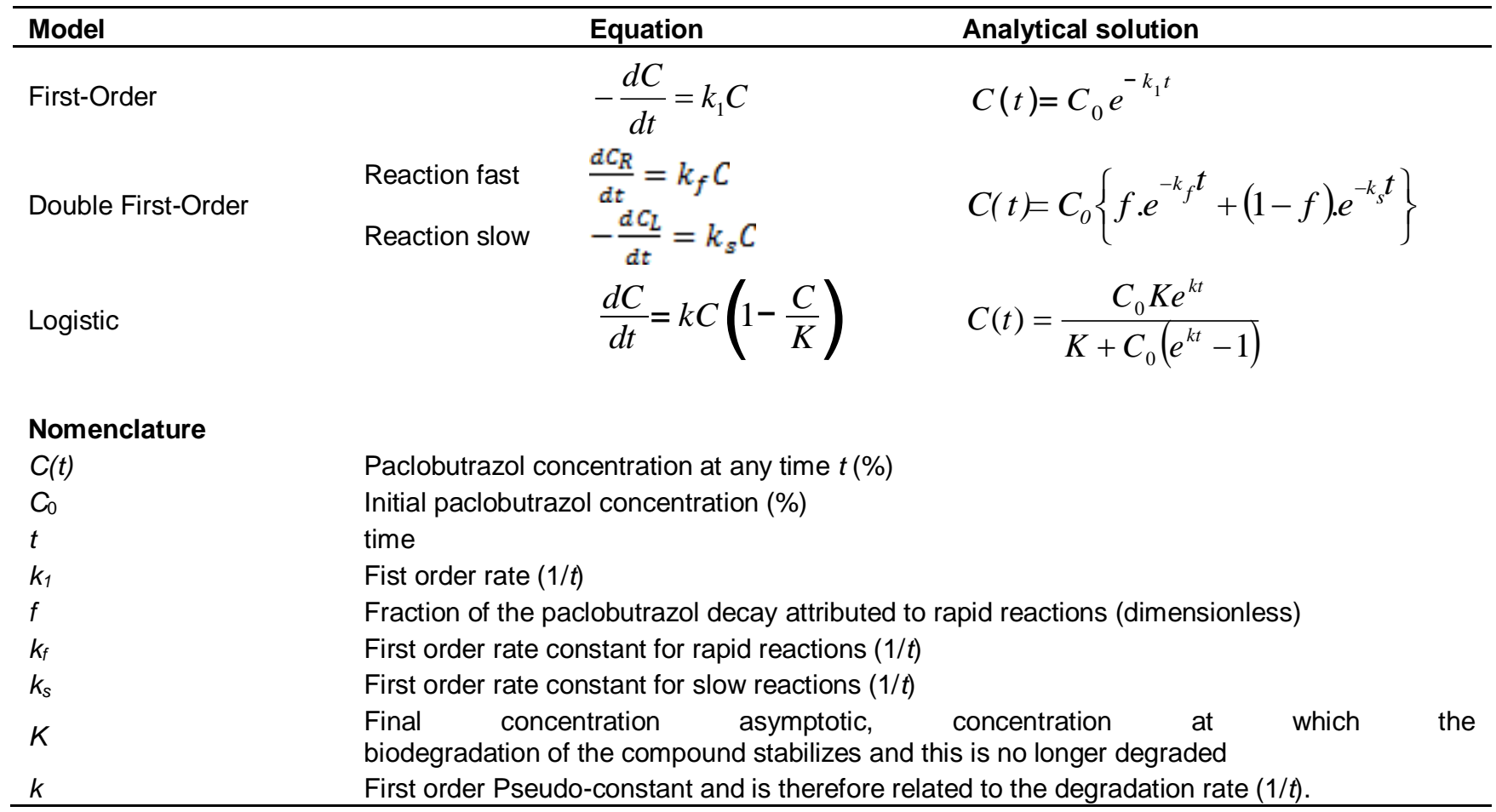

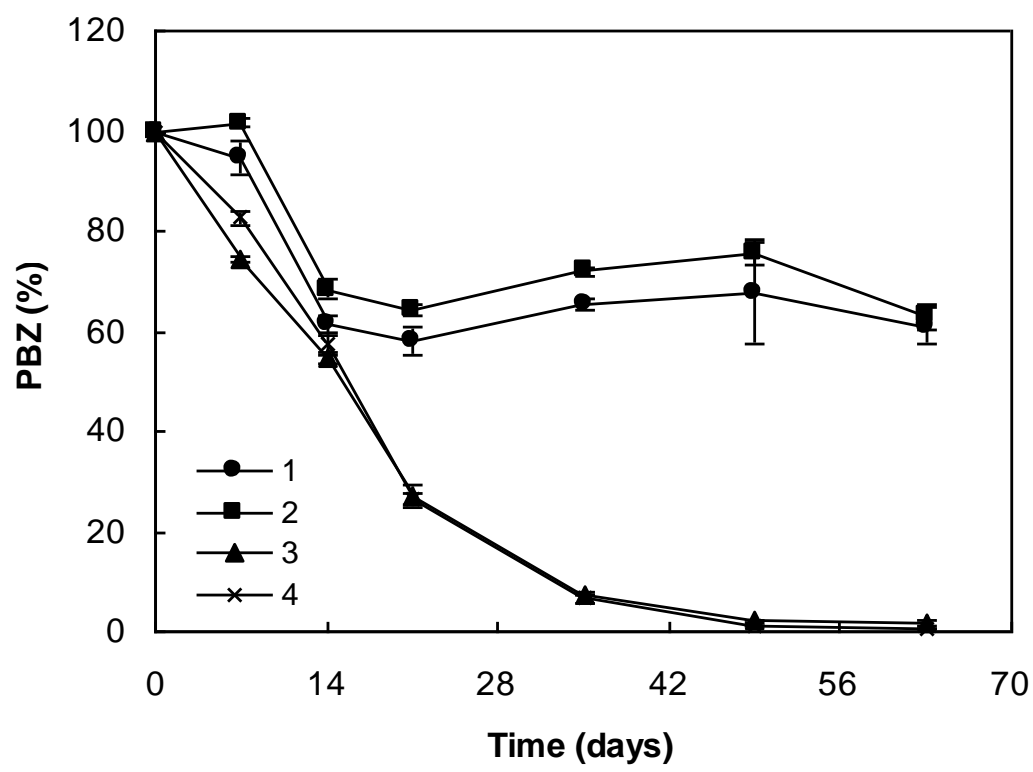

Figure 1. Time course of PBZ. 1, NP-NG; 2, NP-G; 3, P-NG; 4, P-G.

because the native microbiota was not enough to continue the degradation, which was confirmed by lower growth than that obtained in P-G and P-NG (Figure 2).

The biodegradation in NP-G or NP-NG soils were clearly lower than that in P-G or P-NG soils. Biodegradation were not significantly different up to 49 days for experiments in NP-G and NP-NG soils $(F=2.42$ $\alpha=0.55$ ). Similarly, in soil $P-G$ and P-NG soils, the biodegradation were not significantly different up to 14 days $(F=0.09 ; \alpha=0.05)$. Maximum biodegradation occurred in soil with a PBZ application history with 63 days, regardless of the presence of glycerol. Probably 


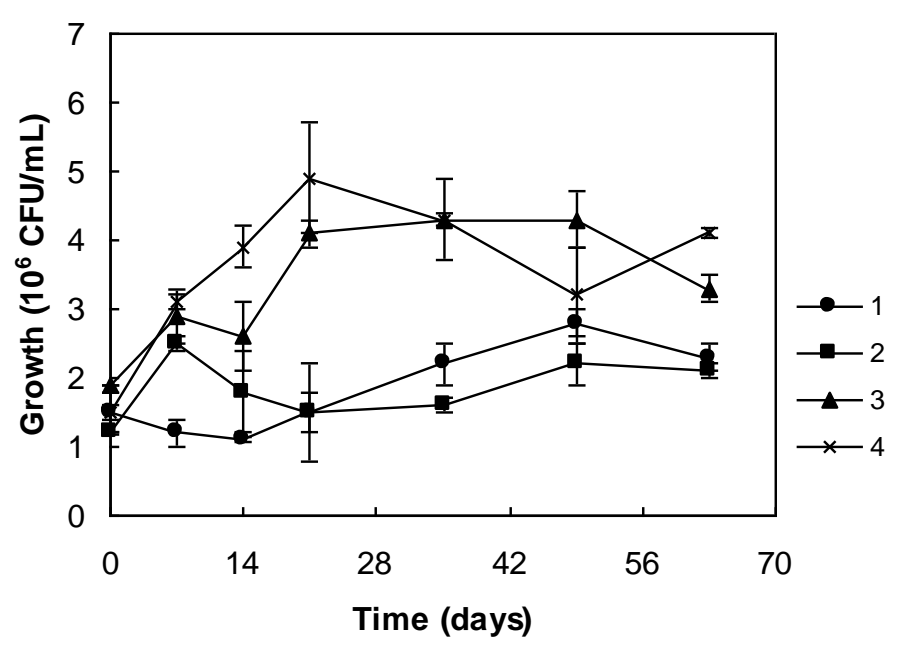

Figure 2. Time course of native microbiota: 1, NP-NG; 2, NP-G; 3, P-NG; 4, P-G.

this was due to the low concentration of glycerol added. Growth was similar; regardless the addition of glycerol, comparing NP-G with NP-NG soils and P-G- with P-NG soils (Figure 2). However, with respect to the soil with and without history, there was higher growth to soil with history (P-G and P-NG).

The PBZ biodegradation kinetics modeling in the experiments without history, and without (NP-NG) and with (NP-G) glycerol are showed in Figure $3 a$ and $b$, respectively. Constants and kinetic parameters of three models (first-order, double first-order kinetic and logistic equation) are found in Table 2. The highest fit for NP-NG followed a double first-order kinetic $(R=0.89)$ and logistic equation ( $R=0.88$ ). On the other hand, only the model of double first-order kinetics was reasonably fitted to the NP-G $(R=0.81)$. The PBZ biodegradation kinetics modeling in the experiments with history, and without ( $P$ $N G$ ) and with (P-G) glycerol are showed in Figure $4 a$ and $b$, respectively. The three models were well fitted to $P-N G$ and $P-G$ soils $(R>0.93)$.

Adjustment of the first-order kinetic model indicates that a single microbial system is involved in biodegradation. On the other hand, when the biodegradation first occurs at a fast reaction and then decays at a slow rate, the double first-order kinetic model is more suitable. As both models were well adjusted at P-NG and P-G soils, the constants of first-order models $\left(k_{1}\right)$ were equal to those of the fast reaction of double first-order kinetic model $\left(k_{f}\right): k_{1}$ $(\mathrm{P}-\mathrm{NG})=0.057 ; \mathrm{k}_{1}(\mathrm{P}-\mathrm{G})=0.054 ; k_{f}(\mathrm{P}-\mathrm{NG})=0.057 ; k_{f}$ $(P-G)=0.054$ (Table 2).

Due to the excellent fit of the first-order kinetic model for P-NG and P-G soils, the constants of the slow reaction were equal to those of the fast reaction of double first-order kinetic model $\left(\mathrm{k}_{1} \_k_{f}=k_{s}\right)$. The first-order kinetic model could not be adjusted for NP-NG or NP-G soils, since that the stabilization of the biodegradation occurred

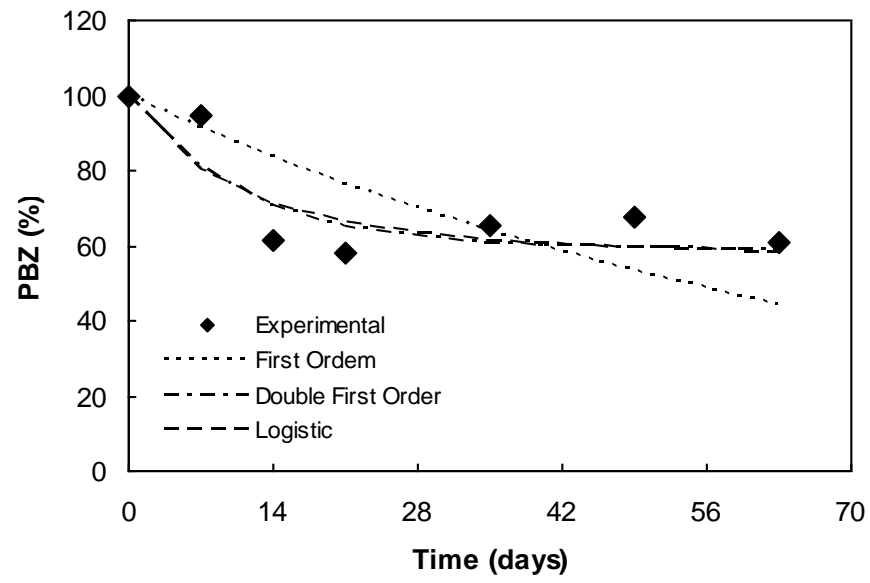

(a)

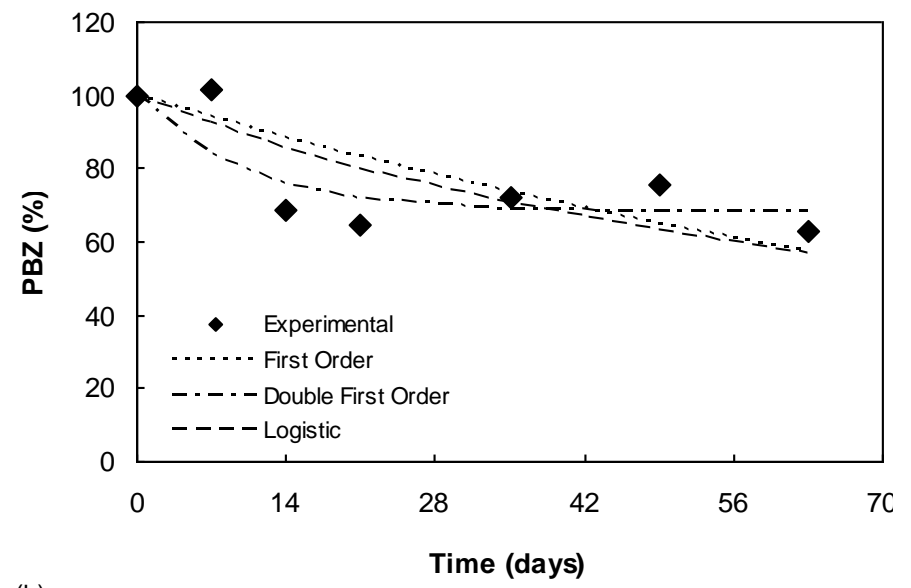

(b)

Figure 3. Modeling kinetics of soil NP-NG (a) and NP-G (b).

in $64 \%$. On the other hand, logistic equation could not be adjusted for only NP-G, since that K was $5.00 .10^{-04}$ and the concentration at which the biodegradation stabilized in this soil was $64 \%$.

PBZ has been shown to be efficient in treating mango trees in semi-arid conditions (Mouco and Albuquerque, 2005). Because it needs to be applied into the soil, it is inconvenient since it remains and effects future planting. Further, it is difficult to determine the dosage for each future use when only empirical methods are used, as there remains residue from the previous cycle of application (Rademacher et al., 2006). No quantification is done nor is it always taken into consideration when deciding the dose. Thus, the applied amount of PBZ in the soil is not always appropriate and the risks of using doses above the recommended are great.

The inflorescences on trees treated with high doses have been very compact (Mouco and Albuquerque, 2005), creating suitable conditions for the incidence of diseases and pests (Winston, 1992), whose control is also hampered by the format of the panicles. Besides the phytosanitary problems, excessive doses of PBZ can 
Table 2. Constants and kinetic parameters of three models applied at biodegradation.

\begin{tabular}{lcccccccccc}
\hline \multirow{2}{*}{ Soils } & \multirow{2}{*}{$\boldsymbol{C}_{\boldsymbol{o}}(\%)$} & \multicolumn{2}{c}{ First order } & \multicolumn{4}{c}{ Double first order } & \multicolumn{2}{c}{ Logistics } \\
\cline { 3 - 11 } & & $\boldsymbol{K}$ & $\mathbf{R}$ & $\boldsymbol{f}$ & $\boldsymbol{k}_{\boldsymbol{f}}$ & $\boldsymbol{k}_{\boldsymbol{s}}$ & $\mathbf{R}$ & $\boldsymbol{K}$ & $\boldsymbol{k}$ & $\mathbf{R}$ \\
\hline NP-NG & 100 & 0.013 & 0.62 & 0.42 & 0.089 & $7.12 .10^{-11}$ & 0.89 & 57.6 & $5.84 .10^{-02}$ & 0.88 \\
NP-G & 100 & 0.009 & 0.51 & 0.32 & 0.103 & $2.69 .10^{-11}$ & 0.81 & $5.00 .10^{-04}$ & $6.09 .10^{-08}$ & 0.60 \\
P-NG & 100 & 0.057 & 0.99 & 1.60 & 0.057 & 0.057 & 0.99 & $9.00 .10^{-04}$ & $9.44 .10^{-07}$ & 0.95 \\
P-G & 100 & 0.054 & 0.98 & 1.00 & 0.054 & 0.054 & 0.98 & $3.77 .10^{-05}$ & $3.72 .10^{-08}$ & 0.93 \\
\hline
\end{tabular}

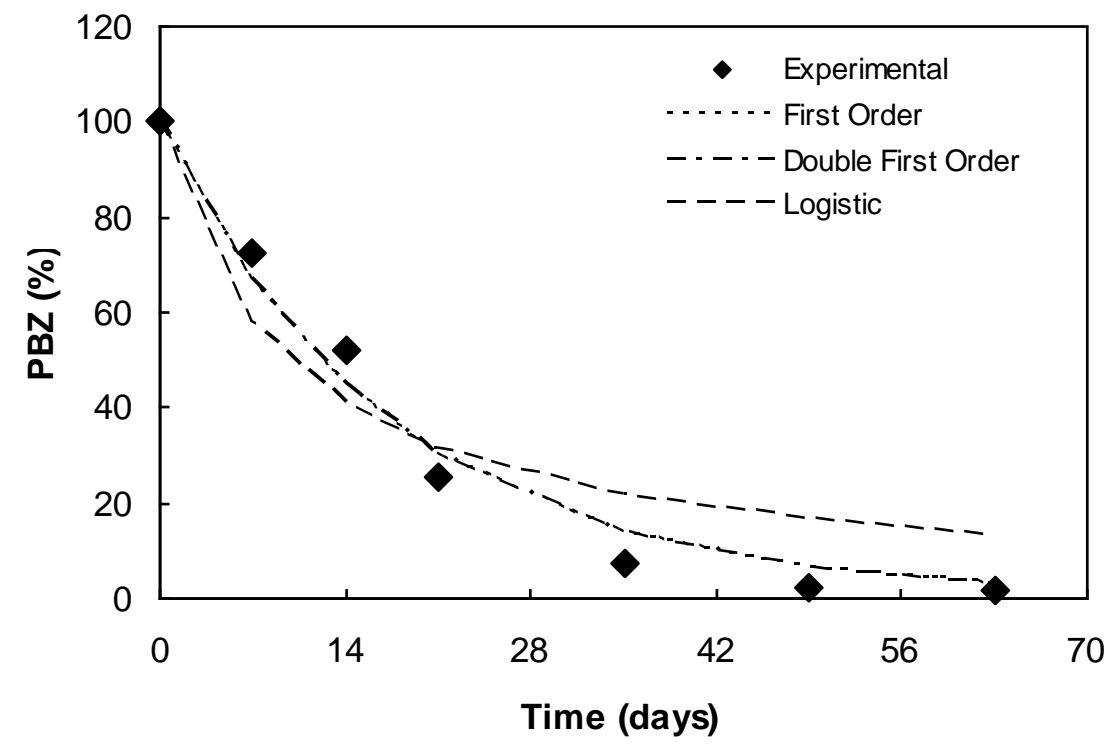

(a)

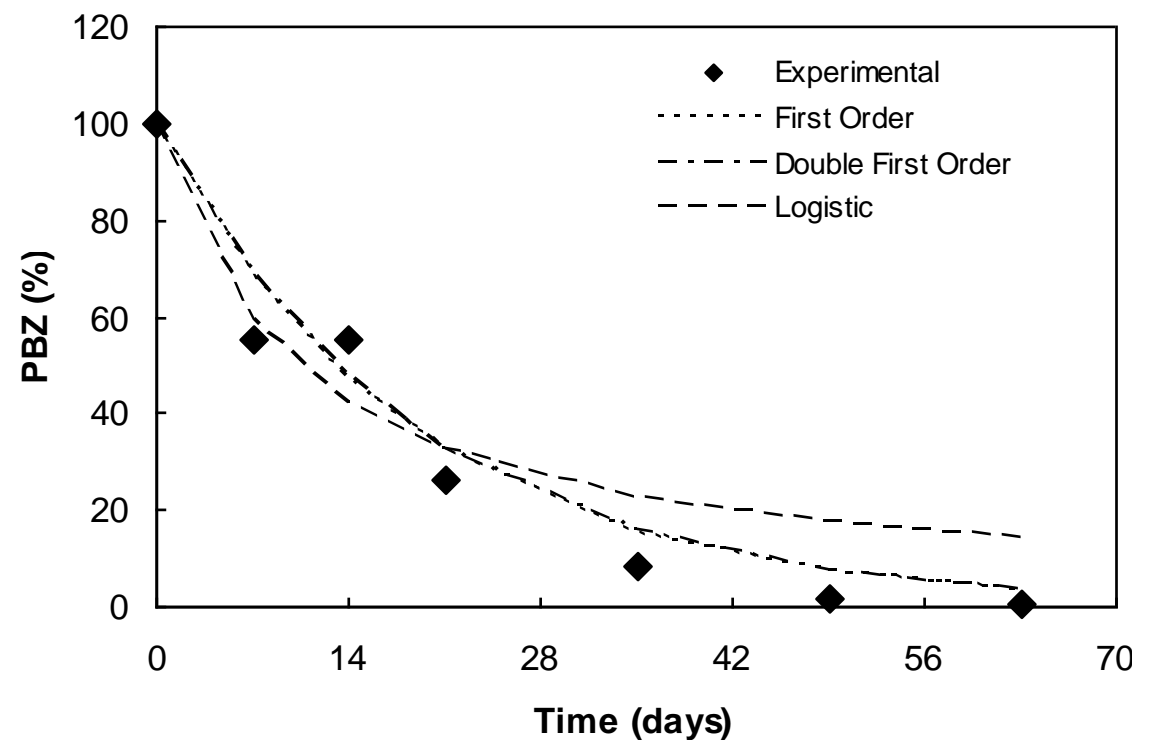

Figure 4. Modeling kinetics of soil P-NG (a) and P-G (b).

inhibit vegetative and floral sprouting longer than desirable, requiring nitrate sprays to stimulate flowering. Thus, in addition to increasing the cost of crop production, for all the reasons that have been mentioned, there is accumulation of chemicals in the ground without knowing the long-term consequences for the production system. 


\section{Conclusion}

Biodegradation of PBZ in unsaturated soils was more efficient when soil samples with a history of application of PBZ were used. We concluded that this soil bacterium is better adapted for the degradation of the compound. Mathematical models can help to identify high levels of toxic substances in soil treated with pesticides and indicate that such substances should be systematically monitored.

\section{Conflict of interests}

The authors have not declared any conflict of interests.

\section{REFERENCES}

Abdelrahman H (2004). Biodegradation of some pesticides by soil microorganisms. In Master thesis. Zagazig University, Agricultural Botany Department.

China M, Kumar S, Kumar S (2004). Sensitivity analysis of biodegradation of soil applied pesticides using a simulation model. Biochem. Eng. J. 19:119-125.

Fujisawa T, Ichise K, Fukushima M, Katagi T, Takimoto $Y$ (2002). Improved uptake models of nonionized pesticides to foliage and seed of crops. J. Agric. Food Chem. 50:532-537.

Gang DC, Clevenger TE, Banerji SK (2003). Modeling chlorine decay in surface water. J Environ Informatics 1:21-27.

Jackson MJ, Line MA, Hasan O (1996). Microbial degradation of a recalcitrant plant growth retardant - paclobutrazol (PP333). Soil Biol. Biochem. 28:1265-1267.
López-Galindo C, Garrido MC, Casanueva JF, Nebot E (2010). Degradation models and ecotoxicity in marine waters of two antifouling compounds: Sodium hypochlorite and an alkylamine surfactant. Sci. Total Environ. 408:1779-1785.

Martins JMF, Mermoud A (1998). Sorption and biodegradation of four nitroaromatic herbicides in mono and multi-solute saturated/ unsaturated soil batch systems. J. Contam. Hydrol. 33: 187-210

Mouco MAC, Albuquerque JAS (2005). Paclobutrazol effect at two mango production cycles. Bragantia 64:219-225.

Rademacher W, Spinelli F, Costa G (2006). Prohexadione-Ca: Modes of action of a multifuncional plant bioregulator for fruit trees. Acta Hortic. 727:97-106.

Srivastav M, Kishor A, Dahuja A, Sharma RR (2010). Effect of paclobutrazol and salinity on ion leakage, proline content and activities of antioxidant enzymes in mango (Mangifera indica L.). Sci. Hortic. 125:785-788.

Varela H, Torresi RM, Gonzalez ER (2002). Aspects related to the use of the quadratic logistic equation in electrochemical processes. Quím. Nova 25:99-106.

Vaz FL, Netto AM, Antonino ACD, Martins JMF, Gouveia ER (2012). Modeling of the kinetics biodegradation of paclobutrazol in two soils of the semiarid northeast Brazil. Quím. Nova 35:77-81.

Winston EC (1992). Evaluation of paclobutrazol on growth, flowering and yield of mango cv. Pride. Australian. J. Exp. Agric. 32:97-104. 